Pedagogía y Saberes No. 46 Universidad Pedagógica Nacional Facultad de Educación. 2017, pp. 65-75

\title{
Carl Gustav Jung y la educación*
}

Artículo de investigación

Carl Gustav Jung and Education

Carl Gustav Jung e a educação 


\title{
Resumen
}

La investigación doctoral de la que deriva este artículo diferenció lo estructural de lo histórico, frente a la formación. Para ello, se revisaron autores que posibilitaran fundamentar esa diferencia. Aunque Carl Jung fue revisado esta lectura no se incluyó en el informe final. Aquí se destacan cinco temas estructurales que señala este autor: el maestro dosifica saberes expuestos y saberes en reserva; no controla su posición frente al otro, ni su auto-representación del oficio; la dimensión instruccional es subordinada, aunque imprescindible; la escuela abre horizonte, en oposición a la familia; la autoridad (no autoritarismo ni falta de autoridad) es necesaria: más allá de los propósitos, permite al aprendiz cambiar su plan vital, involucrando la cultura; y la escuela produce efectos residuales, tramitables en un campo cognitivo donde "hable al alma".

\section{Palabras clave}

Educación (formación), maestro (educador), inconsciente, conciencia, satisfacción.

\begin{abstract}
The PhD research project of which is derived this article, is focused on the differentiation between history and structure regarding training. It was necessary to do some literature review that supported those differences; although Jung was reviewed but not included in the final, five of his main ideas are mentioned: teachers dose reserved and exposed knowledge, they neither control their position in front of the other nor their auto-representation of the profession, instructional dimension is subordinated but essential, the school opens a horizon opposed to family, authority (not authoritarianism or lack of authority) is necessary since, beyond any purpose, it allows the learner to change his vital plan by involving culture in it, and finally, school produces residual effects that can be shown in a cognitive field in which "the soul speaks".
\end{abstract}

\section{Key words}

Education (training), teacher (educator), unconscious, consciousness, satisfaction.

\section{Resumo}

A pesquisa doutoral da qual deriva-se esse artigo diferenciou o estrutural do histórico, frente à formação. Para isso, se revisaram autores que possibilitaram fundamentar essa diferencia. Ainda que Carl Jung foi revisado, essa leitura não foi incluída no informe final. Aqui se destacam cinco temas estruturais: o professor dosifica saberes expostos e saberes em reserva; não controla sua posição frente ao outro nem sua auto representação do oficio; a dimensão instrucional é subordinada, ainda que imprescindível; a escola abre horizonte em oposição à família; a autoridade (não autoritarismo nem falta de autoridade) é necessária: além dos propósitos, permite ao aprendiz mudar seu plano vital involucrando a cultura; e a escola produz efeitos residuais, tramitáveis num campo cognitivo onde "a alma fala".

\section{Palavras chave}

Educação (formação), professor (educador), inconsciente, consciência, satisfação. 


\section{Presentación}

$\mathrm{M}$ uchos de los autores que participaron en la consolidación del psicoanálisis hicieron reflexiones sobre la educación. Durante la investigación conducente a mi tesis doctoral (Bustamante, 2013), hice un análisis pormenorizado de algunos de tales documentos. Entre otros, los de Carl Gustav Jung. Sin embargo, me vi obligado a restringir el corpus y, en consecuencia, la reflexión sobre el trabajo del autor suizo no quedó incluida en el informe final.

A petición de los editores invitados de la revista $P e$ dagogía y Saberes n. ${ }^{\circ}$ 46, dedicada al tema "Pensadores y educación", actualizo la reflexión sobre uno de los textos de Jung: la conferencia titulada "El significado de la psicología analítica para la educación", dictada durante el Congreso Internacional de Educación, celebrado en 1923 en Territet-Montreux (Suiza). Este material no solo tiene interés histórico (de archivo), también permite reflexionar acerca de tópicos de permanente vigencia (estructurales) para pensar lo educativo. El comentario sobre los otros documentos del autor relativos al tema (las conferencias dictadas en los dos siguientes congresos $)^{1}$ desborda la extensión del presente texto.

\section{Introducción}

El título de la conferencia de Jung habla de "psicología analítica" y no de "psicoanálisis". Esto merece una explicación... aunque un junguiano y un seguidor del psicoanálisis freudiano no lo explicarían de la misma manera; y no por "simpatía" por uno u otro modo de entender las cosas. Las disensiones en el campo freudiano pueden enunciarse como diferencias teóricas, o bien como asuntos transferenciales a los que, posteriormente, se les adosa un contenido doctrinal. Y como efecto del tratamiento de lo transferencial, bajo el cobijo de una supuesta discrepancia doctrinal, se pueden tomar caminos que, efectivamente, terminan en diferencias teóricas. Pero, como el psicoanálisis es una teoría de la práctica (y no una teoría, o la aplicación de una teoría), las diferencias no se miden en términos de audacia intelectual, sino como esfuerzos para estar a la altura de la clínica, cuyo destino es lo que finalmente interesa.

Por eso, según Ernest Jones (1953, p. 403), biógrafo de Sigmund Freud, si bien la opinión general juzga las diferencias entre el creador del psicoanálisis y los

1 "Psicología analítica y educación", serie de tres conferencias dictadas durante el Congreso Internacional de Educación del siguiente año (Londres, 1924); así como "El significado de lo inconsciente para la educación individual”, conferencia preparada para el subsiguiente congreso (Heidelberg, 1925). discípulos que se separaron de él basándose en los méritos "objetivos" de sus respectivas teorías, tales diferencias no siempre alcanzan ese estatuto. Fue justamente Freud, en el ensayo "Las resistencias contra el psicoanálisis" (1924a), quien puso a nuestro alcance la idea según la cual las teorías pueden ser blanco de resistencias, en virtud de su capacidad para impugnar el lugar del sujeto. En tal sentido, a Copérnico y a Darwin, más que argumentos, en su momento les oponían protestas, pues sus teorías atentaban contra el lugar que el sujeto se auto-asignaba en el universo y en el panorama de las especies animales, respectivamente. A estas afrentas al narcisismo humano -como las llama-, Freud agrega el caso de su propio trabajo: los reparos a él muchas veces fueron, y son aún, viscerales: pocas veces referidos a los textos, raras veces concebidos con rigor.

La mayoría de los pioneros del psicoanálisis, según Jones (1953, p. 404), no conoció en gran medida su propio inconsciente, por lo cual las resistencias contra el psicoanálisis estaban en ellos latentes, esperando una oportunidad para emerger. Por ejemplo, a propósito de la diferencia entre los psicoanalistas suizos (como Jung) y los vieneses (por ejemplo, Freud), se llegó a considerar sus raíces arias y judías; ante esto, Freud afirmó: "Si estas diferencias se reflejan en la captación de las relaciones objetivas de la ciencia, debe haber algo que no va bien" (citado por Jones, 1953, p. 422), pues, para él, no había ciencia aria o judía.

En 1914, Jung renunció a la Asociación Psicoanalítica Internacional fundada por Freud y empezó a llamar "Psicología analítica" a su trabajo.

\section{Aclaración no pedida...}

En la conferencia, Jung dice (1923, pp. 49-50) haber "abandonado el marco angosto de una técnica de tratamiento médico y su limitación a ciertos presupuestos teóricos", es decir, haber dejado de lado el análisis freudiano y haber pasado a la psicología normal. Al considerar el psicoanálisis como un método médico (1923, p. 49), de un lado, Jung es impreciso, pues si bien Freud y muchos de sus colaboradores (incluyendo al mismo Jung) fueron médicos, eso no determina la especificidad del psicoanálisis; y, de otro lado, "olvida" la historia de ese campo, dada contra la medicina, al punto que la formación de un analista no se corresponde con la del médico (Freud, 1926, p. 230), ni con la del sacerdote ${ }^{2}$ (p. 213).

2 O sea, en la Historia de la sexualidad. La voluntad de poder, Michel Foucault (1976) también lee de manera negligente cuando pone en línea la confesión religiosa y el psicoanálisis. 
Según el pensador suizo, si se trata de medicina, el objeto es lo patológico, mientras él se encarga de la "psicología normal" y, en consecuencia, puede hablar de educación (la cual quedaría fuera del ámbito de lo anormal). Sin embargo, la particularidad de las "patologías" consideradas por Freud es distinta de las de la medicina; justamente, mientras la histeria era vista por los médicos como una puesta en escena, como un fingimiento, él se distingue de ellos por escuchar una verdad en los enunciados de los pacientes. Termina, entonces, proponiendo una nueva manera de entender la especificidad humana, no a partir del cuerpo, aunque se entrelace de forma compleja con él. Esta ya no es una consideración entre los extremos "normal" / "anormal", sino una nueva comprensión de la condición humana, según la cual todos estamos enfermos (Freud, 1917, p. 326).

Así, las referencias de Freud a la educación (1913a, 1913b, 1914, 1925, 1932) pasan progresivamente de la idea de una escuela productora de neurosis, a la idea de sujetos neuróticos por definición ${ }^{3}$; por tanto, la escuela enfrenta indefectiblemente esta característica. Nada tiene de normal el animal humano: le da la espalda a su dotación instintiva, se instala en el lenguaje e inventa ese mundo donde la educación aparece, pues el hombre es la única criatura que ha de ser educada, como apunta Immanuel Kant (1803, p. 29).

Como, según Jung, el psicoanálisis únicamente estudia las ramificaciones del impulso sexual,

[...] mencionarlo sólo estaría justificado si nos hubiéramos propuesto hablar exclusivamente de la psicología sexual del niño. Pero es importante para mí dejar claro que no apruebo esas ideas de acuerdo con las cuales la relación del niño con sus padres, con su maestro, con sus hermanos y con sus condiscípulos hay que explicarla a partir de los inicios inmaduros de la función sexual. (1923, p. 50).

Ante esto, se impone una aclaración: fueron los pacientes quienes le hablaron a Freud de sexo, él no los incitaba a mencionar expresamente ese tema sino, como reza la regla clínica, a dar rienda suelta a las ocurrencias del momento, sin oponer objeciones morales, lógicas o estéticas (algo totalmente ajeno a la "confesión"). Darle su lugar a eso fue su esfuerzo teórico. A sabiendas de las controversias venideras -con mayor razón en esa época-, se opuso a hacer concesiones al moralismo y entendió que "todo

3 " [...] la psicología profunda descubierta por el psicoanálisis era justamente la psicología de la vida anímica normal. Habíamos llegado al mismo resultado que los químicos: las grandes diferencias cualitativas entre los productos se reconducían a variaciones cuantitativas en las proporciones de combinación entre los mismos elementos" (Freud, 1924b, p. 52). aquel que prometa a la humanidad liberarla de las dificultades de lo sexual será aclamado como un héroe, cualesquiera sean las tonterías que se le ocurra decir" (citado por Jones, 1953, p. 423).

La sexualidad no aparece como un "tema" del psicoanálisis, sino más bien como una anomalía lógica gracias a la cual se estructura la posición subjetiva (Lacan, 1972). Por lo tanto, para implicar la sexualidad cuando se estudia el lugar de los sujetos en la educación, no tenemos por qué mencionar la "psicología sexual del niño", como advierte Jung: basta con hablar de la condición humana. La aparición expresa del tema sería apenas una de las posibilidades de tocar el asunto e, incluso, no necesariamente en el sentido del psicoanálisis.

\section{Los componentes}

\section{El éxito de un maestro no depende de su método}

$$
\text { C. G. Jung }
$$

De los puntos tratados por Jung en la conferencia de 1923 he escogido cuatro, vinculados con componentes estructurales de la educación escolar: (1) Cómo hablarle al aprendiz, (2) Qué decirle, (3) Cómo concebirlo y (4) Cómo entender la función del educador.

\section{Cómo hablarle al aprendiz}

De acuerdo con Jung, un maestro de escuela:

[...] nunca debería descargar su conocimiento psicológico directamente sobre el niño, como por desgracia sucede, sino que este conocimiento ha de servirle sobre todo para adoptar una actitud inteligente hacia la vida anímica del niño, pues se trata de un conocimiento para adultos, no para niños, los cuales de momento sólo necesitan cosas elementales. (1923, p. 50).

Esta interesante idea ${ }^{4}$ esboza una definición: el maestro sería un dosificador específico -habría otrosde conocimientos: expone unos ante los estudiantes y mantiene otros en reserva. Aquellos conocimientos que se reserva tendrían funciones delimitadas, como por ejemplo la de fundamentar su actitud hacia el aprendiz. Ahora bien, ¿qué entender por "conocimiento psicológico"? En este contexto, hay al menos tres opciones: un saber disciplinar, un saber procedimental y una doxa. Veamos:

4 Mezclada, no obstante, con el deber-ser: nunca se "debería" descargar sobre el niño su conocimiento psicológico. Pero, ¿por qué ciertos educadores hacen eso (como constata Jung)? La bondad de la consigna no explica por qué es así en algunos casos. 
- Como saber disciplinar, proveniente de, al menos, una disciplina teórica, el "conocimiento psicológico" es explicitable, entendiendo que el maestro se relaciona con la disciplina entre los extremos de pertenecer al campo teórico o de sólo aludir a él, mediado por algún nivel de recontextualización. De hecho, de un tiempo para acá la psicología ha ganado un sitio en la pugna por explicar qué ocurre en el ámbito educativo, de manera que durante la formación docente algo se dispensa de esta teoría ${ }^{5}$. $Y$ es razonable aspirar a que algo como eso no se le diga al estudiante (exceptuando, por supuesto, a quienes buscan profesionalizarse en ese tema).

- Como saber procedimental, el "conocimiento psicológico" no es explicitable y proviene de un atravesamiento subjetivo, de los efectos de la historia vital del maestro. Es, por ejemplo, la actitud que se tiene frente al aprendiz: no proviene de una teoría, sino de la manera como el sujeto mismo tramitó el asunto de su posición como niño, como aprendiz, como sujeto. Es un saber tácito cuyo acceso requiere pasar el trabajo de "hacer consciente lo inconsciente". De ser así, en la historia misma del sujeto estaría al menos parte de la elucidación de su actitud hacia el niño (y, por tanto, de su disposición para el juego entre los saberes expuesto y en reserva). De tal modo, ante un maestro sin la posición garante de la reserva de ciertos saberes, poco sacamos con mostrarle que otra posición es más ventajosa para el proceso educativo (cfr. Tizio, 2002).

- Como doxa, el "conocimiento psicológico" se puede hacer manifiesto. Proviene de una autorepresentación -adobada con elementos disponibles en lo social- sobre el estatuto psicológico de la práctica desencadenada gracias a la mediación docente. En este sentido, ningún maestro estaría desprovisto de un "saber psicológico"; no obstante, este en realidad no fundamenta su acción, y más bien le permite representarse su lugar en ella, independientemente de que esa representación explique algo, y más allá de la medida en que mencione alguna teoría.

Pese a ser saberes excluyentes, el docente los mezclará (lo cual no autoriza a borrar las diferencias entre ellos) en dosis diversas pues, simultáneamente, el maestro (1) es más o menos capaz de dar cuenta de alguna(s) teoría(s) psicológica(s), (2) ha ganado en su vida alguna postura para tramitar lo psicológico con el otro y (3) se auto-representa su labor de

5 No es el momento de establecer cómo lo ha logrado; tampoco se puede inferir de ese hecho-de naturaleza política- la justeza o no de las aproximaciones de la psicología a lo educativo. Lo cierto es que está ahí. algún modo (con participación variable de elementos disponibles en las creencias sociales).

Entonces, el maestro opera desde su posición en relación con esos tres lugares: de entrada, se autoriza a hablarle al estudiante gracias a una elección, hecha con base en la singularidad de su atravesamiento subjetivo -no en vano, no todos quieren ser profesores, $y$ no perseveran todos los que lo han intentado-; por su parte, la modalidad de esta elección establece la distancia que tendrá con el saber disciplinar; y, finalmente, dará a la auto-representación un alcance en función del peso que para él tenga el saber disciplinar. Todo esto, además, está en interacción permanente.

Reflexiones como estas llevaron a Freud (1913b) a pensar, inicialmente, que el educador debía ser instruido sobre la especificidad del niño (pues sus prejuicios al respecto eran desfavorables); pero luego (1925) descartó el saber, a favor de la posición asumida, como resultado del atravesamiento del sujeto maestro en su propia existencia (algo irremplazable luego por teorías). Por ello, pasó de la idea de instruir al maestro, a la idea de psicoanalizarlo ${ }^{6} \ldots$ sin embargo, entendió esta conjetura como irrealizable, pues no podía convertirse en un deber-ser de la formación del docente: mientras analizarse es una decisión de cada uno (no puede ser un imperativo proveniente del Otro), el asunto de cómo formar a los docentes es una decisión social.

Justamente, el texto de 1925 es una introducción a un libro de August Aichhorn, a quien Freud considera como alguien con una sensibilidad particular, gracias a la cual obra, frente a los jóvenes "descarriados", con un talante poco convencional y muy inteligente. No sabía, propiamente; más bien, no podía dejar de hacerlo como lo hacía -si bien, además, quería entenderlo-. Así, si un maestro tiene un conocimiento psicológico y lo descarga directamente sobre el niño, como deplora Jung, no es por estar desinformado, sino por la imposibilidad de obrar con el niño de otra guisa.

Además, Jung considera este conocimiento psicológico como algo para adultos: los niños no serían su destinatario, pues "de momento sólo necesitan cosas elementales". Parece estar pensando más en una teoría, lo que le da la razón: no es para niños. Con todo, aduce algo curioso: mientras los adultos necesitan cosas complejas, los niños necesitan cosas elementales. De acuerdo con esta idea, del niño al adulto habría un desarrollo, un progreso (Jung, 1923, p. 54). De ser así, los procesos educativos tendrían claridad sobre el panorama, pues este iría de un menos a un más.

6 En una posición intermedia estaría Jung (1923, p. 56), quien desaconseja al educador aplicar la teoría de la psicología analítica a los niños, aunque la recomienda para su autoconocimiento, pues su propia educación termina beneficiando a los niños. 
No obstante, el estatuto de sujeto no permite hacer diferenciaciones muy claras entre niños y adultos. La sensación de que la tarea educativa sería distinta en función de la complejidad es solo un acuerdo con una vulgata sobre el desarrollo psicológico, fácilmente objetable: si bien los desafíos del docente varían dependiendo de ciertos grupos etarios, no por ello se hacen menos complejos (podríamos decir que la complejidad varía); de igual forma, un docente no deja de encontrar dificultades por el hecho de trabajar con grupos etarios distintos (la dificultad varía).

Por otra parte, no está fundamentada la idea según la cual los niños necesitan cosas elementales. Si estamos en medio del lenguaje, si cada componente suyo se define por las relaciones sostenidas con los otros, entonces, para poder obtener lo mínimo, requerimos operar con el todo (ejemplo: el niño no aprende a hablar sumando partes elementales para hacer la totalidad compleja de la lengua; al contrario: a partir de la totalidad que infiere, puede desagregar las partes). El mismo Jung ubica una línea divisoria tajante en la vida de un niño cuando "Empieza a decir 'yo'" (1923, pp. 51-53), pues entonces se producen-según él- una continuidad de la conciencia y una memoria (sin dejar de ser una confluencia de fragmentos). $Y$-agregamos nosotros-: porque eso implica situarse en la escena semiótica, no en la escena del "mundo objetivo", pues 'yo' no tiene referente (varía de continuo), solo indica a quien hace uso de la palabra.

Por supuesto, no hablan de lo mismo un niño en el preescolar, un muchacho en la educación media y un joven en la universidad; pero eso no implica que se trate de operaciones más simples o más complejas. Con todo, Jung pone el dedo en la llaga: ¿cómo hablarle al aprendiz?, ¿cuál es el momento oportuno para tocar ciertos temas?, ¿con base en qué decidirlo?, ¿es libre el docente en relación con esto?

\section{Qué decirle al aprendiz}

Para Jung (1923, p. 51), la conciencia es como una isla sobre el mar de lo inconsciente; ambos niveles varían e interactúan todo el tiempo. Sin embargo, luego parece hablar de un crecimiento paulatino de la primera, en detrimento del segundo. En tal sentido, se muestra a favor de apoyar la conciencia mediante la educación: "La escuela no es más que un medio para apoyar adecuadamente el proceso de formación de la conciencia. Y la cultura es la máxima conciencia posible" (Jung, 1923, p. 52). Pero si seguimos su propia definición de lo inconsciente (contenidos que no alcanzan, o han perdido, la intensidad necesaria [p. 51]), ¿podría este reducirse a favor de la conciencia?
Como tiene dos elementos -conciencia e inconsciente $^{7}$-, el autor se ve obligado a tomar partido por la primera. Olvida lo dicho unos renglones atrás: las ocurrencias son representaciones viables gracias a la emergencia de lo inconsciente en la conciencia. Así, la propuesta a favor de la conciencia iría en detrimento de las ocurrencias, de la creatividad. Si los dos conjuntos están en movimiento e interacción permanente, el horizonte de una desaparición de lo inconsciente solo inmovilizaría el "alma" (como la llama Jung). ¿0 nos habla el médico suizo de una posición del sujeto frente a su inconsciente?

Como se sabe, el aparato psíquico en Freud tiene dos tópicas, cada una con tres elementos. Así, por ejemplo, algo no tiene por qué estar consciente para estar disponible; el concepto pre-consciente es justamente ese estado de disponibilidad mediante un esfuerzo sin oposición (lo pre-consciente no ocupa un lugar en lo consciente, ni es inconsciente). Muchos ejercicios escolares -memorizar las tablas de multiplicar, por ejemplo- producen un efecto en este sentido: no buscan hacer consciente nada, sino producir una disponibilidad para otras tareas, estas sí conscientes.

Al optar por la conciencia, nada del orden de lo inconsciente tendría valor desde la perspectiva educativa. No obstante, entendió Freud, la formación no podía reducirse a lo dado por la cultura ("La cultura es la máxima conciencia posible", afirma Jung en la cita en comento). También en ese sentido, Kant (1803, p. 30) hacía una distinción: por un lado, la educación quita -mediante la disciplina-; y, por otro, proporciona -mediante la instrucción-. $\mathrm{Si}$, como el mismo Jung plantea, el poder decir "yo" identifica un momento de inicio, es forzoso formular la constitución de ese estadio no-natural, que implica justamente la extracción de la naturaleza, como condición necesaria para la realización en el sujeto de esa "máxima conciencia posible" que sería la cultura. Desde la misma lógica del autor, no sería dado ampliar la conciencia con la cultura, si no se puede hablar, si el sujeto hablante no se puede ubicar en la enunciación (decir "yo").

Así, la cultura no sería el asunto mismo de la educación, sino apenas una parte de ella. El sujeto hablante, resultado de esa primera extracción hecha sobre el viviente Homo sapiens no es, ni mucho menos, alguien dispuesto a "asimilar" la cultura. El resultado

7 También aparece el yo, pero en este texto lo identifica con la conciencia. Este "yo", con artículo, es distinto de "yo" como primera persona del singular. En español no tenemos dos palabras para hacer la diferencia, como sí ocurre en francés (je y moi, respectivamente), lo que le permite a Lacan (1949) introducir el concepto sujeto, más allá de la tópica freudiana de yo, ello y súper-yo. 
de la primera operación es más bien el de un sujeto de impulsos -los menciona Jung (1923, p. 51) al lado de las ocurrencias, en cuanto irrupción de lo inconsciente en la conciencia-, no alguien dispuesto a ampliar su conciencia con los datos de la cultura.

En tal sentido, solo una apertura previa en su dirección daría lugar a la ampliación de la conciencia con contenidos culturales. Y la escuela nunca deja de ocuparse de esto, aunque lo haga de manera diversa, mientras la persona asciende por sus escalones. O sea: no puede no haber contenidos de la cultura, pero estos solo insertan al campo del Otro, una vez dada la condición de viabilidad de dicha inserción. De tal modo, la escuela no se puede definir meramente como un medio para apoyar el proceso de formación de la conciencia; esa sería (de aceptar tales palabras) apenas una de sus funciones, y no la más importante. Además, para aceptar la idea de la cultura como la máxima conciencia posible habría que entenderla como algo anecdótico, pues basta con cambiar de sociedad o de época para comprobar la variabilidad de la oferta. No puede faltar, por supuesto, una anécdota (unos saberes determinados) en ese punto; tales anécdotas podrían denominarse "máxima conciencia posible", a condición de subrayar su correspondencia con una sociedad concreta. De lo contrario, estaríamos valorando unos conocimientos escolares por fuera de su relación con la hominización y con la opción de hacerse a los saberes funcionales de la época donde hemos sido convocados a existir. Y eso lleva fácilmente a jerarquizar las diferencias culturales: es el caso de Kant (habla de "salvajes" [1803, p. 30]) y del mismo Jung (habla de "razas primitivas" [1923, p. 52]) ${ }^{8}$. Pero mal haríamos en tematizar estos asuntos, a toda vista objetables, pues no son el núcleo de la propuesta de tales autores.

En este punto, entonces, vuelve y señala Jung algo fundamental: el "sentido" de la instrucción no estriba propiamente en lo dicho -aunque sea imprescindible decirlo-, sino en la modificación que eso entraña para el sujeto. Y esa cornucopia de la cultura está situada en otro lugar (en el lugar del Otro). De ahí que la escuela sea un ámbito fundamental.

\section{Cómo se concibe al aprendiz}

Para Jung (1923, p. 52), los niños sin escuela, abandonados a sí mismos, serían inconscientes: vivirían en un estado primitivo, salvaje; su inteligencia sería instintiva; permanecerían ignorantes, sin conciencia de

8 Freud (1912), por su parte, iguala a los llamados "primitivos" con los llamados "civilizados", pues se ven obligados a tramitar los mismos asuntos, pese a las diferencias culturales. sí mismos ni del mundo. El niño va de un estado inconsciente (semejante al del animal) hacia la conciencia, primero primitiva y luego -poco a poco- civilizada.

De nuevo, para entender la educación, Jung cuenta con la oposición inconsciente/conciencia, la cual tiende a definirse en términos de contenidos culturales: su ausencia equivale a "ignorancia", mientras su presencia equivale a "civilización"; en ella, "inconsciente" participa de dos estatutos muy diferentes: el de la animalidad y el que convive con una "conciencia primitiva". Si consideramos a los llamados "niños salvajes", nos toca darle la razón a Jung: la ausencia de escuela -para seguir su razonamiento- no permitiría sacar al Homo sapiens pequeño de su estado de animalidad (pero, ¿es eso "inconsciencia"?).

Ahora bien, con más categorías, podemos hacer otras distinciones y la descripción se complica: no se trata tanto de ausencia de escuela como de ausencia de vínculo humano. El caso de "niño salvaje" estudiado por Jean Itard (1801/1806), muy bien documentado 9 , muestra el fracaso rotundo del propósito instructivo cuando se intenta llevar a cabo con alguien sin vínculo con el lenguaje, dada una prolongada ausencia de relación con seres hablantes.

Si un niño habla, no importa qué lengua, es capaz de aprender; y un niño capaz de aprender, aprende, no importa de qué contenidos se trate ${ }^{10}$. Solo en la última fase, donde se registra la presencia de una sociedad interesada en reproducir sus anécdotas, es donde surge el asunto de los contenidos (de la instrucción, propiamente dicha), al parecer realzado por Jung. Para el psicoanálisis, los contenidos son un correlato -imprescindible- de algo producido en términos estructurales, formales, no de contenido (cfr. Antelo, 2005). Si nos obnubilamos por los contenidos y sus transformaciones, nunca lo veremos.

Con la caracterización hecha por Jung (1923, pp. 53-54) del escolar, se perfilan las dificultades y los constituyentes propios de la educación. Según él, el niño sería una hechura del otro; en tal sentido, cuando entra al colegio, a los seis años, es solo un producto de sus padres y no puede afirmar su individualidad; incluso, manifestaciones suyas -como la testarudez y la desobediencia-no expresan tanto su propio ser, como las influencias perturbadoras de sus padres.

Esta tipificación nos pone ante una aporía: ciertamente, el niño, como ser hablante, como candidato a aprendiz, es en gran medida un producto del otro. Sin

9 Llevado finamente al cine por François Truffaut en El niño salvaje (1970).

10 No dejan de resonar en este punto las Meditaciones de Descartes: pienso -no importa qué-, luego existo. 
el otro, no sería ni hablante, ni candidato a aprendiz. Ahora bien, el proceso de hominización, ¿funciona perfectamente?, ¿solo da lugar a lo planeado? Si así fuera, el sujeto sería inimputable (y sabemos la dificultad inherente a ese terreno): si el otro lo determinara radicalmente, no existiría la responsabilidad, y la libertad ni siquiera sería pensable. Entonces, ¿de dónde nos autorizamos para pedirle cuentas al sujeto, aun desde muy pequeño? El sujeto es quien responde, en ambos sentidos (Miller, 1988): como interlocutor y como responsable de sus actos. Ante esto, pueden aparecer argumentos -reputados como éticos- según los cuales la dimensión de la responsabilidad es uno más de los asuntos otorgados por el otro a escala de los contenidos (por eso, en la escuela se habla de "valores"). Pero, ¿cómo saber si la reflexión ética es un punto de partida y no una respuesta? Como punto de partida, la vemos en los buenos propósitos, dados en la cantilena -moralista o no-, entendidos como condición de la acción... pero, como se dice popularmente, "de buenas intenciones está empedrado el camino al infierno".

En consecuencia, la labor de la escuela depende del estatuto otorgado a estos temas. Por eso, Jung concluye al respecto: "La escuela desempeña una función muy importante en esta lucha de liberación, pues es el primer entorno que el niño encuentra fuera de la familia" [1923, p. 54]. Era de esperarse, pues ante tal determinismo del otro (en primer lugar, la familia), las opciones solo estarían fuera del campo de ese otro. Si la familia ejerce un poder que esclaviza, la escuela luce como liberadora. Y, efectivamente, la escuela para un niño no es indiferente. Podría ser -y con mayor razón cuando Jung dictó su conferencia- su primer entorno extrafamiliar. Ahora bien, ¿en qué consiste tal importancia?, ¿en realidad son tan poderosas la familia -para lo negativo- y la escuela -para lo positivo-?

De entrada, para ponerlo en términos de una "lucha de liberación" -como hace Jung- el niño sería un luchador por la libertad; sin embargo, la descripción de nuestro autor, abreviada y comentada antes, no lo había ubicado en esos términos, sino más bien en términos de desarrollo, de progreso. De lo dicho en la conferencia, no se puede concluir la existencia de una lucha del niño por liberarse. ¿O se trata de una "liberación" paradójica en la medida en que la entrega otro? (así hablamos de la autonomía en la escuela: como algo otorgado por el otro, lo cual contradice la idea misma de "autonomía").

Contemplemos la idea de liberación: ¿no se daría en medio del lenguaje, condición de posibilidad de la relación del sujeto con los otros... de los cuales pretendería liberarse?; ¿hay alternativa para el sujeto por fuera del lenguaje? Cambiar unas ideas por otras no nos libera de las ideas; cambiar unas formas de sujeción por otras, no nos libera de la sujeción ${ }^{11}$. Si no hay retorno a la condición animal, una vez conquistada la condición de ser hablante, es irrenunciable aquello que verdaderamente nos ata. Los referentes no están brindados en términos de situaciones diferentes por las cuales optar.

Si definimos al niño con los elementos en disputa (los efectos de haber advenido en el campo del otro versus la expectativa de tener una "psique individual autónoma", como la nombra Jung [1923, p. 53]), aunque de una manera un poco más compleja, se abren alternativas para entender la labor de la escuela: si ella es el primer entorno que el niño encuentra fuera de la familia, ¿en qué radica su importancia?, ¿por qué al respecto Jung trae a cuento la "libertad"? Si no lo pensamos en relación con propósitos, con ideales como el de "libertad", podríamos encontrar algunas razones.

El niño ha consolidado un régimen de satisfacción en el marco de la familia, grupo privilegiado de personas; si ahí no es autónomo, es por conveniencia propia. Entonces, cuando llega a la escuela, no le está dado encontrar la satisfacción tal como siempre lo ha hecho (aunque la busque), ni en relación con las mismas personas (cuya presencia reclama). Por lo tanto, ¡no quiere liberarse!... al contrario. Entonces, ¿qué tiene esta primera experiencia escolar para considerarla importante?, ¿no sería más bien motivo de "frustración" para el niño? Las nuevas condiciones lo presionan a dejar el ámbito cerrado de una satisfacción más o menos autista. De ahora en más, puede aprender a satisfacerse involucrando al otro (cfr. Millot, 1979). De esta forma, consigue, en algún momento, virar su atención hacia el saber; vía privilegiada para construir una sociedad. Entre querer patear el balón y festejar un gol del equipo, sin haber participado directamente de la jugada... se han introducido nuevos elementos: el paso por el otro como condición de satisfacción y un nuevo objeto (menos ligado a lo sensible) para alcanzarla.

Con la llegada a la escuela también tiene lugar la inyección de tiempo: mientras la satisfacción tiende a ser inmediata, la escuela introduce poco a poco, aquí y allá (a propósito de las tareas, de los recreos, de la alimentación, de las idas al baño...), en intensidades distintas, un intervalo antes de la satisfacción. La necesidad de trabajar, durante un tiempo, para obtener una gratificación, posibilita construir relaciones sociales, proyectos... a diferencia de la satisfacción que tiende -como el ámbito familiar-a reducir el lapso de tiempo entre la demanda del niño y la satisfacción.

11 De nuevo, resuena Descartes: considera un Dios engañador pero, al final, se ve obligado a ponerlo como condición de su ejercicio meditativo. 
Como se ve, el asunto no es propiamente liberarse, sino, más bien, atarse de un modo distinto, introducir nuevas modalidades y objetos de satisfacción, un aplazamiento y un trabajo. Sin la aquiescencia del sujeto, nada de esto es factible. Paradójicamente, solo en una sujeción tolerada por el sujeto pueden configurarse otra modalidad de goce. Por otro lado, una "libertad" incondicional lleva a lo peor, según nos dijo el mismo Jung: el niño sería inconsciente, salvaje e ignorante.

Entonces, el autor apunta a algo fundamental en este sentido: "Un vínculo fuerte con los padres es un obstáculo para la adaptación posterior al mundo. La persona en crecimiento está destinada para el mundo, no ha de ser siempre un hijo de sus padres" (Jung, 1923, p. 55). Más que "dar libertad", entonces, la educación amplía el horizonte del sujeto.

\section{Cómo entender la función del educador}

Según Jung (1923, p. 55), el educador es quien logra "trasladar al niño al mundo y completar así la educación de sus padres". La formación inicial en la familia produce efectos no esperados: o le quita al niño la oportunidad para adquirir responsabilidad individual, con lo cual solo le queda la opción de la dependencia o de una independencia por caminos retorcidos; o bien, por dejar de ejercer la autoridad necesaria, le impide "adaptarse correctamente" al mundo. En estas circunstancias, más que conducir metódicamente unas materias (ipero a condición de hacerlo!), el maestro influencia al niño, sin oprimirlo, representando ante él una autoridad abridora de horizonte (Jung, 1923, p. 55). Todo ello a propósito de unas materias, claro está, pero estas no serían el asunto central de la educación.

Acá hay una propuesta muy sugestiva: el compromiso propio del vínculo familiar impide visualizar los efectos de su acción. No es que la familia "no sepa" sobre el papel de la autoridad y las consecuencias de ejercerla de una u otra manera, sino que "entiende" a través del vínculo afectivo (no forzosamente positivo). Incluso, puede estar enterada del tema... sin embargo, entendery asumir son distintos (Sercovich, 1977). Si en la familia las cosas fueran de otra índole (ideales), la labor del maestro sería enseñar metódicamente unas materias, pues ya contaría con sujetos dispuestos a aprender, producto de la familia. Desafortunadamente, no es el caso. Por eso, de acuerdo con el médico suizo, aunque la enseñanza no responda a "exigencias modernas", sí es productiva cuando se da una "buena relación" maestro/aprendiz (1923, p. 56)... no es "dar amor", no es algo deliberado; se da sólo si produce ciertos efectos: cuando la autoridad produce disposición para entrar al mundo y no inhibición, dependencia o síntoma. Esta sería la parte más importante, pues una vez dada, instruirse es posible; pero al contrario no funciona, pues esta disposición es condición necesaria para la instrucción. Por eso, Jung no está interesado en la "actualidad" del método, sino en el papel efectivo del educador. 0 sea: por un lado, la presencia de una persona-y no cualquiera- es imprescindible; por otro, la formación que podría dispensar la escuela es algo estructural, aunque inevitablemente la escuela esté ubicada en época y lugar.

En medios educativos se piensa en hacer "reformas" consistentes en prescribir el buen ejercicio de la autoridad (o su eliminación, como se cree hacer hoy)... Pero no es algo producto de la voluntad, del método o de los contenidos. La actitud, afirma Jung (1923, p. 55), "no se puede establecer artificialmente, ni aun con la mejor intención". ¡La formación no se da en el nivel de las intenciones!; solo puede producirse cuando el maestro tiene ciertas características: es alguien más o menos "ubicado" (no se ha visto obligado a optar por caminos "retorcidos", como dijo Jung), cumple con su deber, es un buen ciudadano: "El método más perfecto no sirve de nada si la persona que lo aplica no se encuentra por encima de él, gracias al valor de su personalidad" (1923, p. 55).

¿Se trata, entonces, de "ser un buen ejemplo", como está tentado a concluir Jung (1923, p. 55)? No precisamente, pero sí tiene relación: ¿por qué alguien habría de "tomar como ejemplo" a otro? Si la educación psicológica real es obra de la personalidad del maestro (Jung, 1923, p. 55), es porque el aprendiz decide orientarse por lo que cree ver en él, pues tiene algo. En esa dirección, podríamos desbrozar un poco el camino: el concepto del maestro como "buen ciudadano" no viene de la idea sosa según la cual los niños aprendan del buen ejemplo; si aquel está ubicado es porque en él han ocurrido ciertos elementos... recordemos: no es algo imitable, algo de lo que se pueda hacer semblante. El educador está causado de cierto modo y eso se convierte en un enigma para el aprendiz, quien, en consecuencia, se vuelve hacia él para tratar de responder.

Ahora bien, se nos atraviesa una implicación de lo afirmado: ¿cómo se constituyó ese maestro sin sufrir las carencias de la formación inicial en la familia? No es alguien de una familia excepcional, pues tales carencias son indefectibles: ninguna familia se salva (acá naufraga la intención inicial de Jung de no referirse a lo anormal, sino a lo normal). Podríamos pensar lo siguiente: el educador pasó por la escuela y quiere para otros esa experiencia que desagrega del vínculo familiar y da lugar al vínculo cultural. De tal manera, la escuela se hace necesaria... no por razones históricas, sino por razones estructurales. 
La clave parece estar en la exterioridad de la escuela en relación con la familia. Ya habíamos anotado todas las implicaciones derivadas del hecho de verse obligado a buscar la satisfacción en condiciones distintas...

Finalmente, solo se puede sostener esta función a condición de desarrollar activamente la cultura, de no ser su portador pasivo. De lo contrario, "empezará a corregir en los niños los errores que no corrige en sí mismo" (Jung, 1923, p. 57). Notable idea, derivada de los conceptos, no de consignas políticas (como la de la educación permanente): la función docente no está garantizada; si el maestro trabaja en ella es -indirectamente- por desarrollar en acto la cultura, lo cual no es un producto de los buenos propósitos sino de las alternativas reales; el deseo en relación con otra cosa tributa a sostener la función educativa del docente. La segunda parte de la idea no resulta tan clara: ¿Por qué, de no ser así, empezará a corregir en los aprendices los errores que no corrige en sí mismo? ¿Hablamos de errores de contenido? Y, si no fuera de esa forma, ¿en qué sentido desarrollar activamente la cultura evitaría sus errores?... sobre todo, advertidos de las dos dimensiones en juego: la de la postura frente a lo inconsciente y la del aumento de la conciencia con base en la cultura.

\section{Lo residual}

Hasta acá, la formación parecía culminar en la labor escolar: si bien la acción familiar deja restos, la escuela se encargaría de tramitarlos. Pero no, pues, para Jung (1923, p. 56), "alguien que ha ido al colegio y a la universidad, todavía no está educado". Es decir, aun ese lugar donde dejábamos el ámbito familiar, inhibidor de la independencia, causante de caminos no-sociales... puede dejar cosas pendientes: “... se acaba la educación como si las personas ya estuvieran terminadas. La solución de las demás cuestiones complejísimas de la vida queda a cargo del individuo, con su opinión y su ignorancia" (Jung, 1923, p. 56). Se desgaja la conclusión: el adulto es educable, aunque es menos influenciable, pues

[...] ha perdido la extraordinaria plasticidad de la psique infantil, tiene una voluntad propia, convicciones propias, una conciencia de sí mismo más o menos determinada [...]. Se ve como el portador de la cultura actual. Por tanto, no está dispuesto a reconocer (como un niño) un educador por encima de sí. (Jung, 1923, p. 57).

Y está bien así -agrega Jung-; de lo contrario, se trataría de un retroceso a un estado infantil de dependencia. El adulto, en consecuencia, se auto-educa mediante dos procedimientos: (1) el estudio crítico de sus acciones y (2) el enjuiciamiento de sus acciones por otros. Pero el primero está sometido al prejuicio y el segundo puede ser erróneo o no lo aceptamos. ¿Hay algún referente objetivo en relación con lo psíquico? De cara a la conciencia, lo psíquico parece arbitrario y caprichoso; en cambio, frente a lo inconsciente, estamos ante productos -como el sueño- que presentan objetivamente nuestra verdad, pues no están hechos conscientemente $y$, de ese modo, no podemos engañarnos (Jung, 1923, p. 58). Pero, para poder hacer hablar ese material, es forzoso conocer la teoría. Y, entonces, llegamos al final: nunca estamos acabados, si queremos trabajar un poco en eso, recurrimos al que sabe:

Tenemos que educar a unos adultos a los que no podemos dirigir (como a los niños) mediante la autoridad, cuya vida es tan individual que ni el consejero más competente podría indicarles el camino adecuado para ellos. Por tanto, tenemos que hacer hablar al alma de la persona para que ésta entienda desde dentro cómo es. (Jung, 1923, p. 61).

Como la plasticidad de la psique -como dice Jungse pierde de forma paulatina, la sociedad lleva a los sujetos a la escuela cuando están pequeños. Pero, humanos como somos, no estamos exentos de los efectos residuales. Hay algo ingobernable en nosotros (Freud retomaba el chiste según el cual gobernar y educar son tareas imposibles). Ese ingobernable se trata en la familia... pero deja sus restos; se trata en la escuela... pero deja sus restos. Para eso están los que sugieren la condición humana de una pugna insalvable entre impulsos y sociedad: para tratar el resto, para "hacer hablar al alma", como dice Jung.

\section{Conclusión}

El saber no puede, como una pátina social, ungir a todos por igual. Si así fuera, el proceso educativo tendría otras características y, prácticamente, carecería de dificultades. La escuela se dirige a grupos, pero sus efectos solo se producen uno por uno -en virtud de la presencia de un Otro- y son, hasta cierto punto, impredecibles. Si logra la instrucción, es gracias a conseguir una economía de satisfacción en relación con el saber; si alguien, a su vez, tiene una economía de satisfacción ligada con la cultura y quiere eso para los aprendices. Por tal razón, "El éxito de un maestro no depende de su método" (Jung, 1923, p. 56). El método -pero también tendríamos que decirlo a propósito de los contenidos y de los propósitos- acapara la atención cuando cesa de estar aquello de lo que sí depende el éxito del educador. 


\section{Referencias}

Antelo, E. (2005). Notas sobre la (incalculable) experiencia de educar. En: Educar: ese acto político. Buenos Aires: Del Estante Editorial.

Bustamante, G. (2013). Sujeto, sentido y formación. Bogotá: San Pablo-Universidad Pedagógica Nacional.

Descartes, R. (1641/2011). Meditaciones metafísicas. Madrid: Gredos.

Foucault, M. (1976/1977). Historia de la sexualidad. 1. La voluntad de saber. México: Siglo XxI.

Freud, S. (1913a/1990). Introducción a Oskar Pfister, El método psicoanalítico. En: Obras completas (vol. XII). Buenos Aires: Amorrortu.

Freud, S. (1913b/1990). El interés por el psicoanálisis. En: Obras completas (vol. XIII). Buenos Aires: Amorrortu.

Freud, S. (1914/1990). Sobre la psicología del colegial. En: Obras completas (vol. XIII). Buenos Aires: Amorrortu.

Freud, S. (1917/1990). Los caminos de la formación de síntoma. En: Obras completas (vol. XVI). Buenos Aires: Amorrortu.

Freud, S. (1924a/1990). Las resistencias contra el psicoanálisis. En: Obras completas (vol. XIX). Buenos Aires: Amorrortu.

Freud, S. (1924b/1990). Presentación autobiográfica. En: Obras completas (vol. xx). Buenos Aires: Amorrortu.

Freud, S. (1925/1990). "Prólogo" (al libro Juventud descarriada, de August Aichhorn). En: Obras completas (vol. XIX). Buenos Aires: Amorrortu).
Freud, S. (1926/1990). ¿Pueden los legos ejercer el análisis? Diálogos con un juez imparcial. En: Obras completas (vol. xx). Buenos Aires: Amorrortu,

Freud, S. (1932/1990). Conferencia 34. En: Nuevas conferencias de introducción al psicoanálisis. Obras completas (vol. XXII). Buenos Aires: Amorrortu.

Itard, J. (1801/1806/1982). Victor de l'Aveyron. Madrid: Alianza.

Jones, E. (1953/2003). Vida y obra de Sigmund Freud. Barcelona: Anagrama.

Jung, C. G. (1923/2010). El significado de la psicología analítica para la educación. En: Sobre el desarrollo de la personalidad. Obra completa (vol. 17). Madrid: Trotta.

Kant, I. (1803/2003). Pedagogía. Madrid: Akal.

Lacan, J. (1949/1984). El estadio del espejo como formador de la función del yo tal como se nos revela en la experiencia psicoanalítica. En: Escritos I. México: Siglo XXI.

Lacan, J. (1972/1981). Aun. Seminario 20. Barcelona: Paidós.

Miller, J. (1988/2006). Salud mental y orden público. En: Introducción a la clínica lacaniana. Barcelona: RBA.

Millot, C. (1979/1982). Freud anti-pedagogo. Barcelona: Paidós.

Sercovich, A. (1977). El discurso y los fantasmas. En: El discurso, el psiquismo y el registro imaginario: ensayos semióticos. Buenos Aires: Nueva Visión.

Tizio, H. (2002/2005). Actualidad en la conexión psicoanálisis-pedagogía. En: Seminario de otoño. Córdoba: Centro de Investigación y Estudios Clínicos. 